\title{
First-Principle Computation of Relaxation Times in Semiconductors for Low and High Electric Fields
}

\author{
Simon C. Brugger ${ }^{1}$ and Andreas Schenk ${ }^{1,2}$ \\ ${ }^{1}$ Integrated Systems Laboratory, Swiss Fed. Inst. of Technology, Gloriastr. 35, CH-8092 Zürich, Switzerland \\ 2 Synopsys Switzerland LLC, Affolternstrasse 52, CH-8050 Zürich, Switzerland \\ e-mail: brugger@iis.ee.ethz.ch, Tel: +41 44632 2348, Fax +41 446321194
}

\begin{abstract}
In this paper we present a method to compute exact relaxation times for any moments of the Boltzmann equation. The method is valid for any electric field and is particularly useful in the case of vanishingly small field intensities. After a theoretical explanation, the method is applied to a full-band model for electrons and holes in silicon. A strong dependence of the relaxation times on the doping concentration is shown, which is in contradictions to popular beliefs.
\end{abstract}

\section{INTRODUCTION}

Over the past 50 years different approximations have been used to compute transport parameters (mobility, diffusion constant, energy relaxation time, ...) for the drift-diffusion and hydrodynamic transport models (see e.g. [1], [2]). To our knowledge no exact expression has ever been derived for the relaxation times needed for the even moments (energy, energy squared, squared norm of the velocity, ...) of the transport models close to thermodynamic equilibrium. Only an approximation formula for the energy relaxation time was given by Jungemann et al. [3].

In this paper we will present a general scheme and a general formula to compute these relaxation times. This scheme holds for any Boltzmann equation (BE) and does not require specific approximations besides those already present in the BE itself. We will then give results for silicon using a full band structure and compare them with the approximation formula of Ref. [3].

\section{THEORY}

\section{A. Introduction}

Using the Monte Carlo method, the relaxation times in bulk material are usually computed using the formula (see e.g. [3]):

$$
\tau_{g}=-\frac{\int g(k)\left(f(k)-f_{e q}(k) \frac{n}{n_{e q}}\right) d^{3} k}{\iint g(k) S\left(k, k^{\prime}\right) f\left(k^{\prime}\right) d^{3} k^{\prime} d^{3} k},
$$

where $f$ is the solution of the stationary BE, $f_{e q}$ is the equilibrium distribution, $n$ (resp. $n_{e q}$ ) is the integral of $f$ (resp. $f_{e q}$ ) on the $k$-space, and $g$ is the moment for which the relaxation time is needed. This formula, however, becomes problematic in the case of small electric fields especially for an even function $g$.

We found a general method and a general discretization scheme that can be applied at least to any semiconductor
BE (linear and nonlinear) to compute moments of the inverse scattering operator (ISO) $S_{g}^{-1}$ with the vital property:

$$
\left\langle S_{g}^{-1}|S| f\right\rangle=\langle g \mid f\rangle-\left\langle g \mid f_{e q}\right\rangle \frac{n}{n_{e q}} .
$$

Here, $S$ is the scattering operator (collision term in the rhs of the BE), $f_{e q}$ the equilibrium distribution function, and $f$ and $g$ are arbitrary continuous functions. Based on this theory, another formula for the relaxation times can be derived. By multiplying the space-homogeneous BE:

$$
-\frac{q}{\hbar} \vec{E} \cdot \nabla_{k} f=S|f\rangle
$$

with $g$ and $S_{g}^{-1}$ and integrating one obtains

$$
\begin{aligned}
& -\frac{q}{\hbar} \vec{E} \cdot \int\left(\nabla_{k} f\right) g(k) d^{3} k= \\
& \qquad \int g(k) S\left(k, k^{\prime}\right) f\left(k^{\prime}\right) d^{3} k^{\prime} d^{3} k
\end{aligned}
$$

$$
\begin{aligned}
& -\frac{q}{\hbar} \vec{E} \cdot \int\left(\nabla_{k} f\right) S_{g}^{-1}(k) d^{3} k= \\
& \qquad g(k)\left(f(k)-f_{e q}(k) \frac{n}{n_{e q}}\right) d^{3} k .
\end{aligned}
$$

Inserting the rhs of (4) and (5) in (1) gives:

$$
\tau_{g}=-\frac{\vec{n} \cdot \int\left(\nabla_{k} f\right) S_{g}^{-1}(k) d^{3} k}{\vec{n} \cdot \int\left(\nabla_{k} f\right) g(k) d^{3} k},
$$

where $\vec{n}$ is the vector pointing in the direction of the electric field. Note that (6) can be easily extended to the spaceinhomogeneous BE. Using (6) allows to compute exact relaxation times particularly in the limit of a vanishing electric field. For high electric fields the Monte Carlo method can be used to evaluate either (1) or (6).

\section{B. The problem of small electric fields}

For small electric fields the distribution function $f$ can be written as:

$$
f(k)=f_{e q}(k)\left(1+\frac{q}{k_{B} T_{e q}} \vec{E} \cdot S_{\vec{v}}^{-1}(k)\right)+O\left(E^{2}\right),
$$

where $S_{\vec{v}}^{-1}$ is the vector whose i-th component is the $(\vec{v})_{i^{-}}$ moment of the ISO. The important point for the following 
is that using standard methods, the $O\left(E^{2}\right)$ terms cannot be computed.

Inserting (7) into (1) gives:

$$
\begin{aligned}
& \tau_{g}= \\
& -\frac{\int g(k)\left(f_{e q}(k) \vec{E} \cdot S_{\vec{v}}^{-1}(k)+O\left(E^{2}\right)\right) d^{3} k}{\iint g(k) S\left(k, k^{\prime}\right)\left(f_{e q}\left(k^{\prime}\right) \vec{E} \cdot S_{\vec{v}}^{-1}\left(k^{\prime}\right)+O\left(E^{2}\right)\right) d^{3} k^{\prime} d^{3} k}
\end{aligned}
$$

For any system with an even band structure $(\epsilon(k)=\epsilon(-k))$, the function $f_{e q}(k) \vec{E} \cdot S_{\vec{v}}^{-1}(k)$ is odd, because $S_{\vec{v}}^{-1}$ has by construction the same symmetry properties as $\vec{v}$. Thus, if the function $g$ is an even function, (8) reduces to

$$
\tau_{g}=-\frac{\int g(k) O\left(E^{2}\right) d^{3} k}{\iint g(k) S\left(k, k^{\prime}\right) O\left(E^{2}\right) d^{3} k^{\prime} d^{3} k} .
$$

Because $O\left(E^{2}\right)$ cannot be computed in the general case using standard methods, (9) cannot be evaluated. Therefore, formula (1) is unusable at all for even functions $g$ in the limit of a vanishing electric field.

\section{The heated Maxwellian ansatz}

Jungemann et al.[3] tackled the problem by using a heated Maxwellian as ansatz for $f$ :

$$
f(k):=e^{-\frac{\epsilon(k)}{k_{B} T}},
$$

and computed $\tau_{g}$ in the limit $T \rightarrow T_{e q}$. Plugging this into (1) leads to

$$
\tau_{g}=-\frac{\int \epsilon(k) f_{e q}(k)\left(g(k)-\frac{g_{e q}}{n_{e q}}\right) d^{3} k}{\iint g(k) S\left(k, k^{\prime}\right) \epsilon\left(k^{\prime}\right) f_{e q}\left(k^{\prime}\right) d^{3} k^{\prime} d^{3} k},
$$

where $g_{e q}:=\left\langle g \mid f_{e q}\right\rangle$.

The scattering operator $S\left(k, k^{\prime}\right)$ can be formally separated in one elastic part $S\left(k, k^{\prime}\right)_{\text {elastic }}$ and one inelastic part $S\left(k, k^{\prime}\right)_{\text {inelastic. }}$ The elastic part has the property:

$$
\int S\left(k, k^{\prime}\right)_{\text {elastic }} h\left(\epsilon\left(k^{\prime}\right)\right) d^{3} k^{\prime}=0,
$$

for all function $h$ of the energy. Thus, the denominator of (11) does not depend on the elastic scattering. This is unfortunate, because in most models the impurity scattering is an elastic process.

Therefore, the heated Maxwellian ansatz comes off badly in the case of silicon, in particular because contributions from the impurity scattering automatically drop out, which yields relaxation times independent of the doping concentration.

\section{Exact solution}

Inserting (7) in (6) gives:

$$
\begin{aligned}
& \tau_{g}= \\
& -\frac{\vec{n} \cdot \int\left(\nabla_{k}\left(f_{e q}\left(1+\frac{q}{k_{B} T_{e q}} \vec{E} \cdot S_{\vec{v}}^{-1}\right)\right)\right) S_{g}^{-1} d^{3} k+O\left(E^{2}\right)}{\vec{n} \cdot \int\left(\nabla_{k}\left(f_{e q}\left(1+\frac{q}{k_{B} T_{e q}} \vec{E} \cdot S_{\vec{v}}^{-1}\right)\right)\right) g d^{3} k+O\left(E^{2}\right)}
\end{aligned}
$$

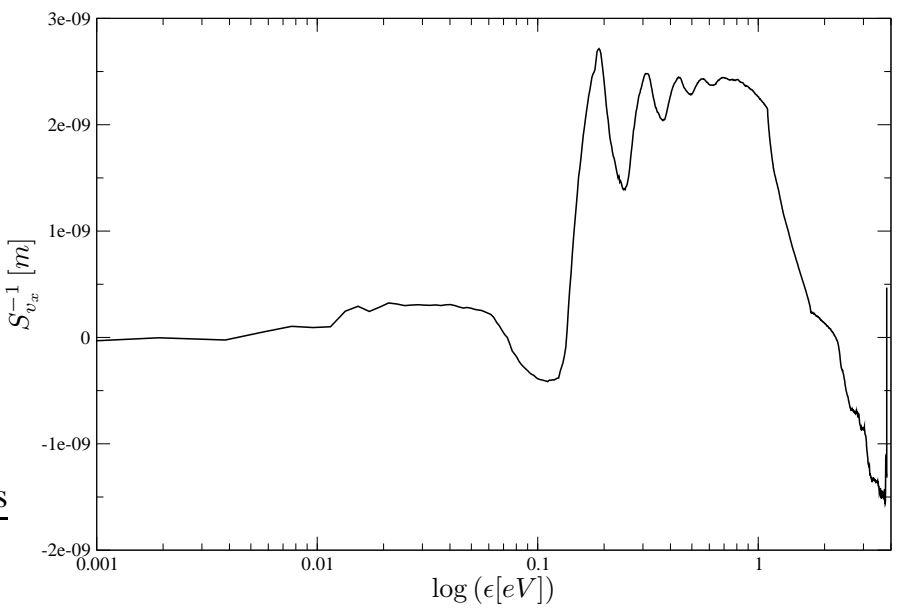

Fig. 1. $S_{v_{x}}^{-1}$ averaged on an iso-energy surface as a function of energy in the first band and the valley along the $\langle 100\rangle$ direction for electrons

Considering again an even function $g$ and neglecting the $O\left(E^{2}\right)$ terms, (13) reduces to:

$$
\tau_{g}=-\frac{\vec{n} \cdot \int \nabla_{k}\left(f_{e q}(k) \vec{n} \cdot S_{\vec{v}}^{-1}(k)\right) S_{g}^{-1}(k) d^{3} k}{\vec{n} \cdot \int\left(\nabla_{k}\left(f_{e q}(k) \vec{n} \cdot S_{\vec{v}}^{-1}(k)\right) g(k) d^{3} k\right.},
$$

because $S_{g}^{-1}(k)$ has by construction the same symmetry properties as $g(k)$.

As (14) is independent of $E$ there is no problem anymore to evaluate $\tau_{g}$ in the limit $E \rightarrow 0$. One can easily show that if $\epsilon(k)$ is an even function, than (14) does also not depend on $\vec{n}$, i.e. the direction of the electric field.

Thus, we proved that the knowledge of $S_{g}^{-1}(k)$ allows one to compute relaxation times for all moments of the Boltzmann equation, even in the limit of a vanishing electric field.

\section{RESULTS}

In this part we will focus on the low field case. The theory has been applied to full-band silicon, to electrons and holes, and to different lattice temperatures and doping concentrations. We used the full-band structure and the scattering models as described in [4] and [5].

To give the reader an idea about the shape of the $S_{g}^{-1} \mathrm{~s}$, two examples have been chosen. Fig. 1 shows the x-component of the average of the function $S_{\vec{v}}^{-1}$ on an iso-energy surface (see eq. (14)) as a function of energy in the first band and the valley along the $\langle 100\rangle$ direction for electrons. Fig 2 shows the $\mathrm{x}$-component of the function $\frac{d S_{\epsilon}^{-1}}{d \epsilon}$ as a function energy in the first band and the valley along the $\langle 100\rangle$ direction for electrons. This function appears in (14) in the numerator, if one performs an integration by parts.

Using (14), we computed the relaxation times for the energy $\langle\epsilon\rangle$, the energy squared $\left\langle\epsilon^{2}\right\rangle$, for $\left\langle v^{2}\right\rangle$ and $\left\langle v^{4}\right\rangle$ as they are needed e.g. in the six-moments model of Grasser et al. [6].

The bold lines in Figs. 3-10 show the results from the exact computation, whereas the thin lines are computed using the ansatz from Jungemann et al. [3]. 


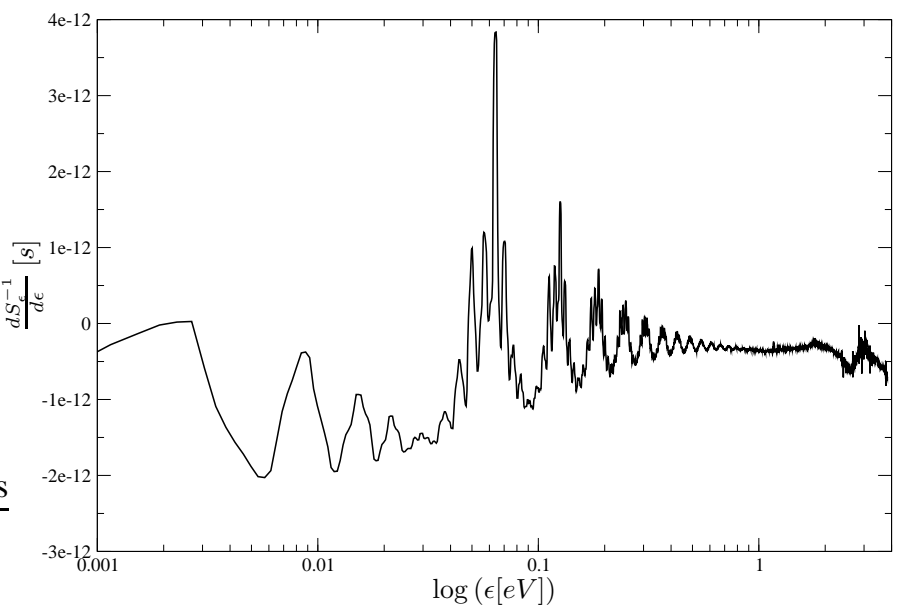

Fig. 2. $\frac{d S_{\epsilon}^{-1}}{d \epsilon}$ as a function of energy in the first band and the valley along the $\langle 100\rangle$ direction for electrons

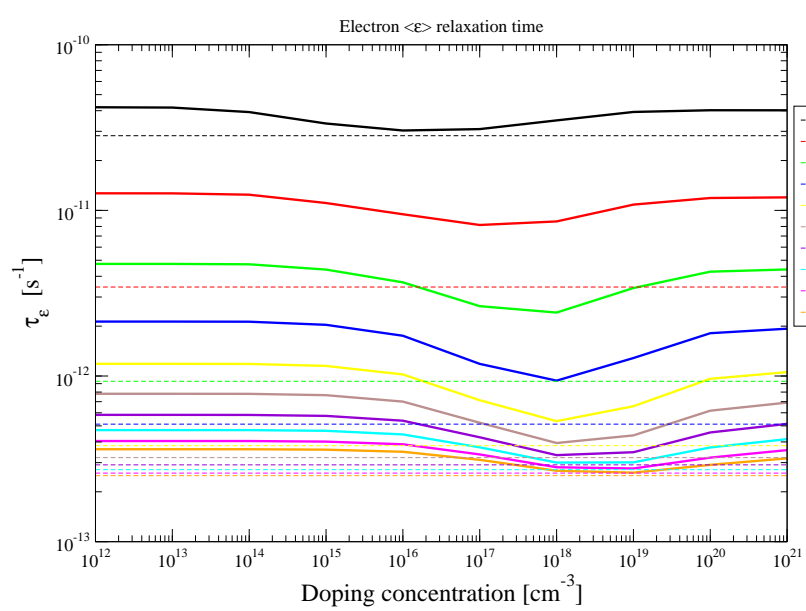

Fig. 3. Energy relaxation time for electrons

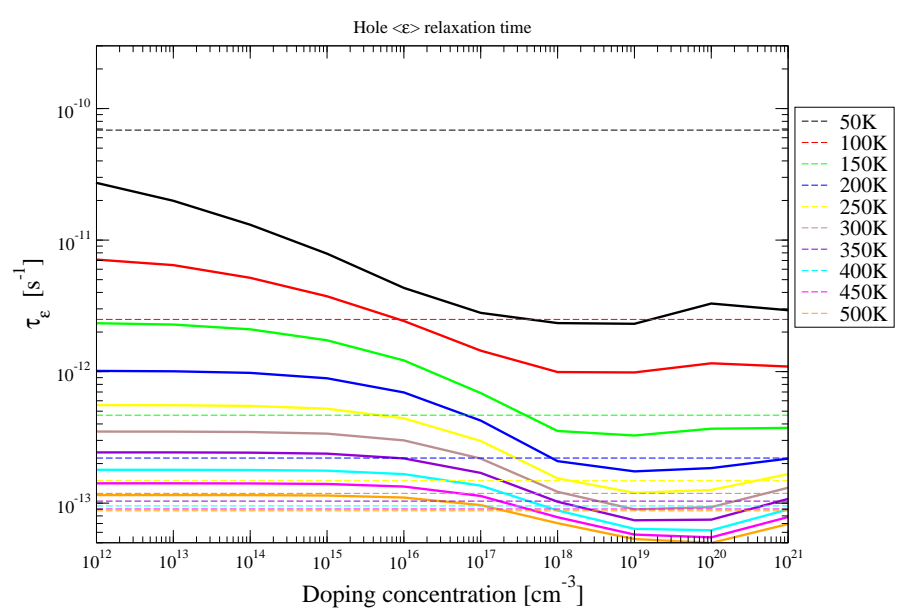

Fig. 4. Energy relaxation time for holes

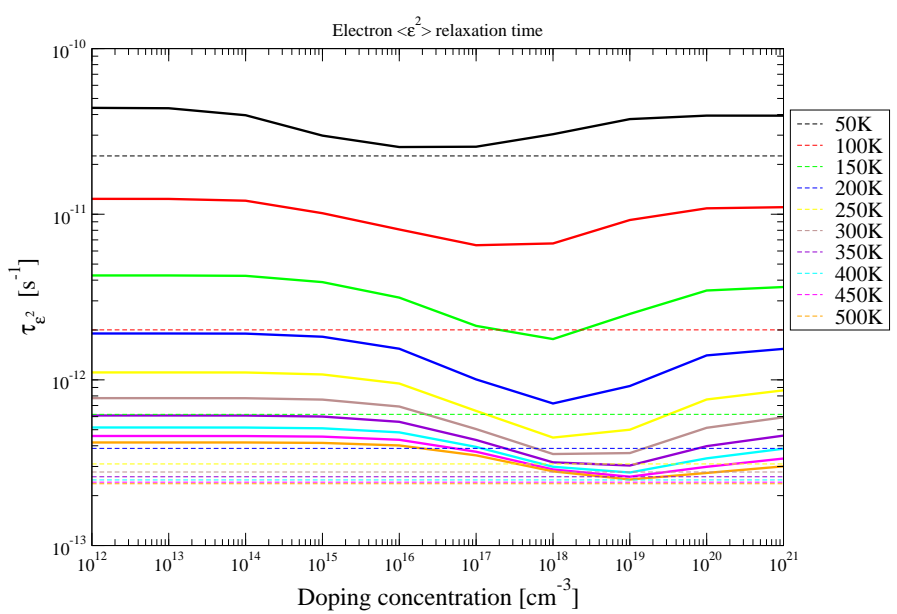

Fig. 5. Energy squared relaxation time for electrons

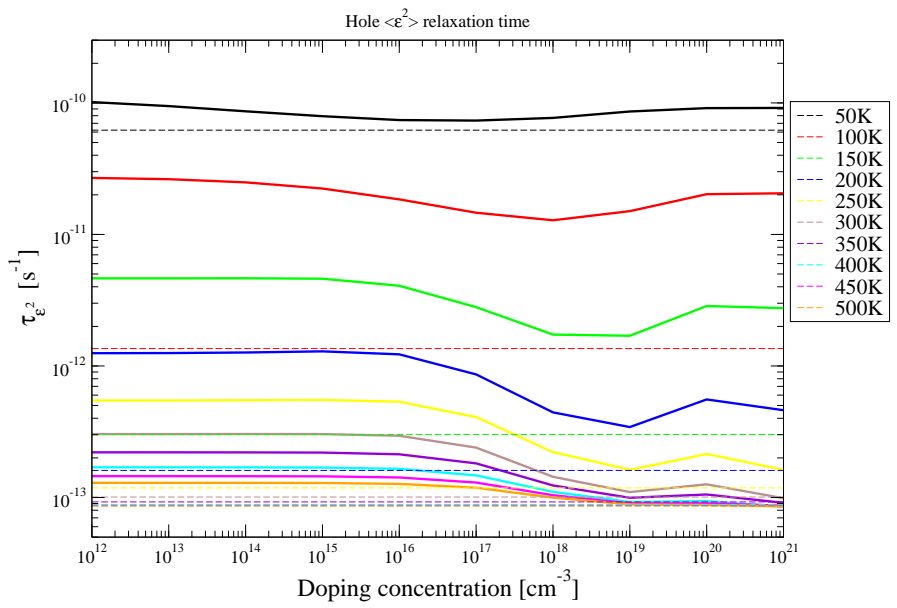

Fig. 6. Energy squared relaxation time for holes

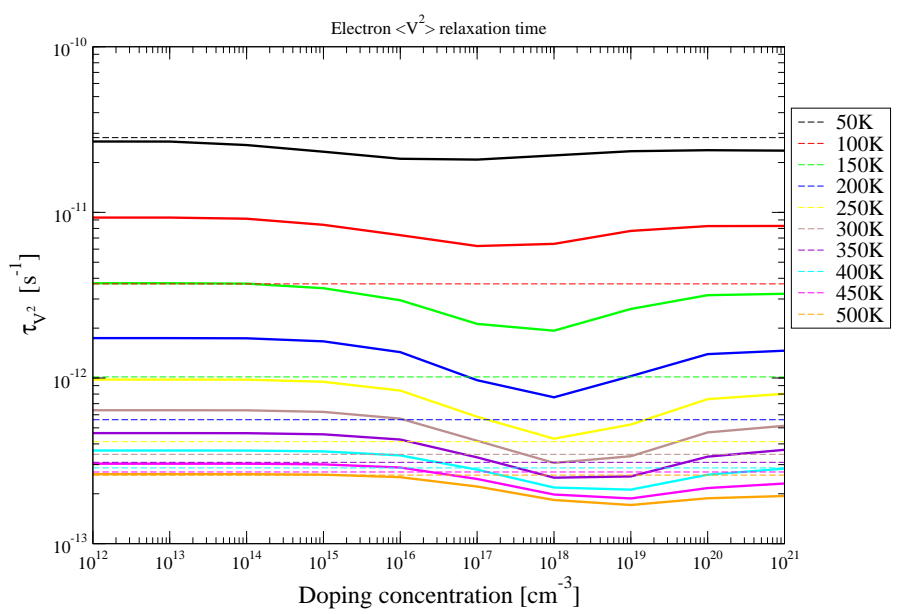

Fig. 7. Velocity squared relaxation time for electrons 


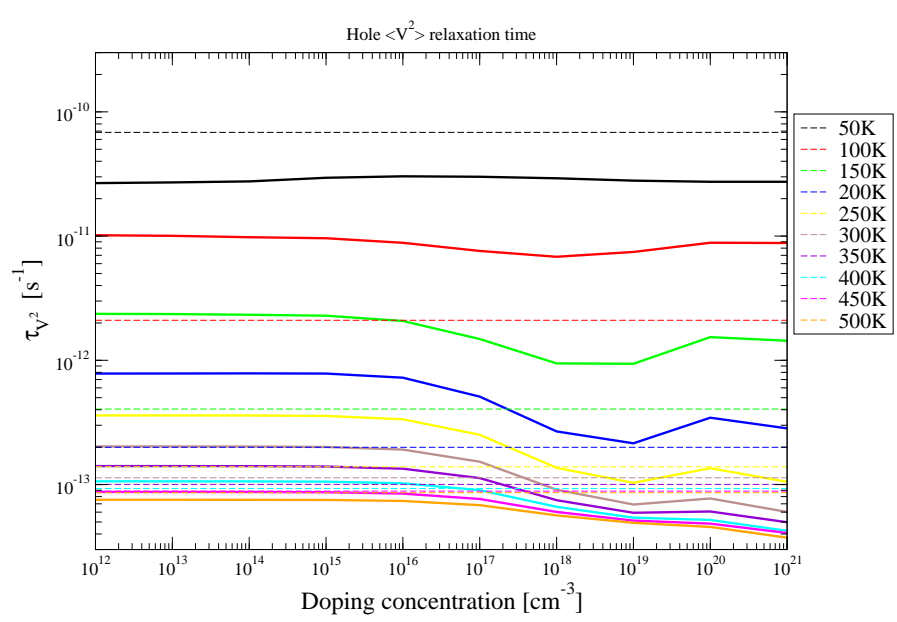

Fig. 8. Velocity squared relaxation time for holes

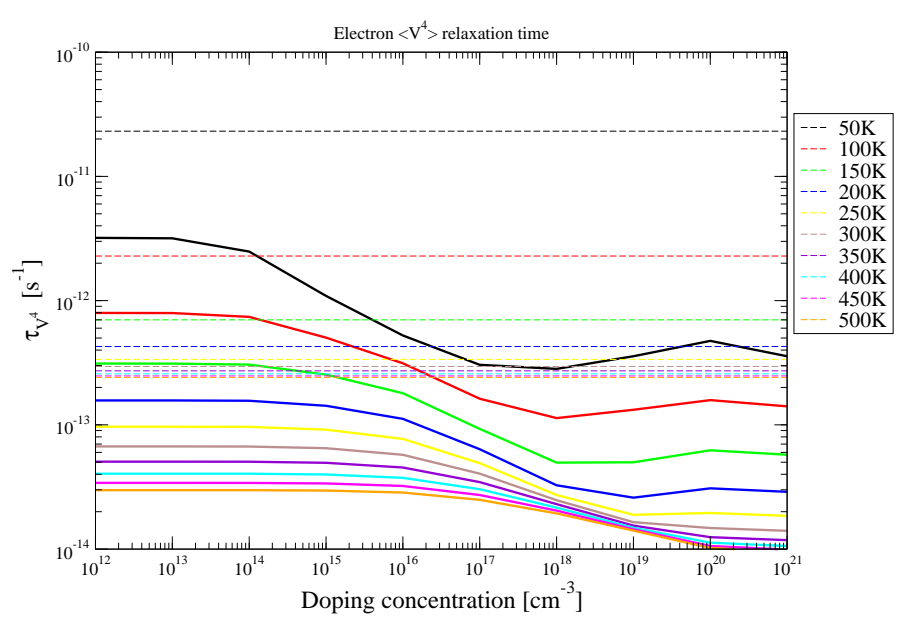

Fig. 9. Velocity to the fourth power relaxation time for electrons

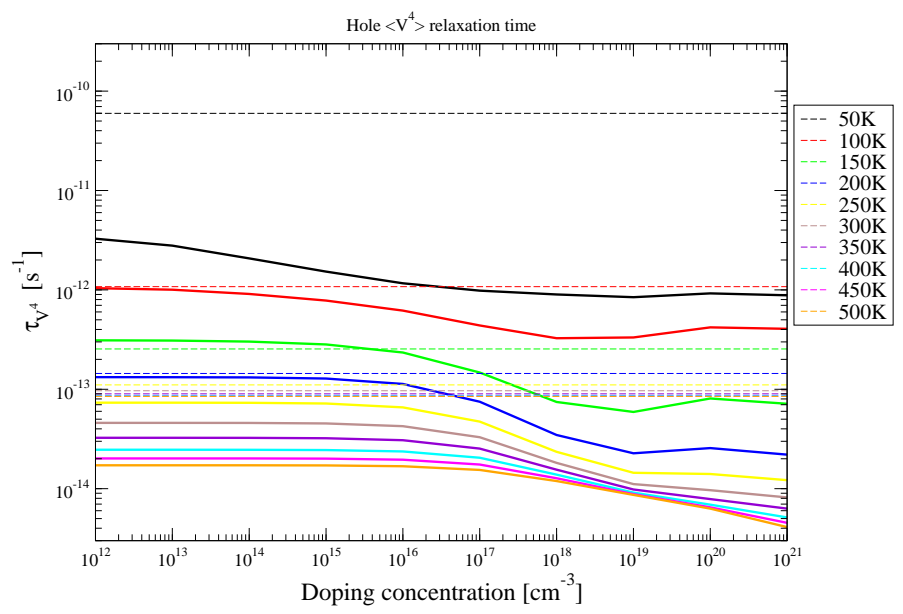

Fig. 10. Velocity to the fourth power relaxation time for holes
It seems that in general the Maxwellian ansatz (11) is rather poor and gives inaccurate results. One also observes a quite strong dependence on doping concentration which can be explained by a detailed inspection of the functions in (6). The exact low-field relaxation times allow to reproduce the Nyquist theorem more precisely than with the usual approximations. In general, the relaxation times show a non-negligible dependence on doping concentration. This is in contradiction to what can be found in the literature (see e.g. [1]).

\section{CONCLUSION}

A method has been found to compute exact relaxation times. This method is especially important for low electric fields and even functions $g$. It can be applied to any semiconductor BE. Discrepancies between the usual approximations found in the literature (e.g. [1], [6] or [3]) and the exact results prove the utility of our method.

\section{ACKNOWLEDGEMENT}

The authors are grateful for the financial support by Fujitsu Laboratories Ltd.

\section{REFERENCES}

[1] T. Grasser, T. Tang, H. Kosina, and S. Selberherr, "A review of hydrodynamic and energy-transport models for semiconductor device simulation," Proc. of the IEEE, vol. 91, no. 2, pp. 251-274, 1999.

[2] O. Madelung, Introduction to Solid-State Theory, ser. Solid-State Sciences. Berlin Heidelberg New York: Springer, 1996.

[3] C. Jungemann and B. Meinerzhagen, Hierarchical Device Simulation. The Monte-Carlo Perspective, ser. Computational Microelectronics. Wien, New York: Springer, 2003.

[4] F. Bufler, Y. Asahi, H. Yoshimura, C. Zechner, A. Schenk, and W. Fichtner, "Monte carlo simulation and measurement of nanoscale n-mosfets," IEEE Transaction on Electron Devices, vol. 50, no. 2, 2003.

[5] F. Bufler, A. Schenk, and W. Fichtner, "Simplifed model for inelastic acoustic phonon scattering of holes in si and ge," Journal of Applied Physics, vol. 90, pp. 2626-2628, 2001.

[6] T. Grasser, H. Kosina, M. Gritsch, and S. Selberherr, "Using six moments of boltzmann's transport equation for device simulation," Journal of Applied Physics, vol. 90, no. 5, pp. 2389-2396, 2001. 